\title{
MS031.P03
}

\section{Silver nanoparticle sulfidation kinetics across all length scales}

Andrew John Allen ${ }^{1}$, Fan Zhang ${ }^{1}$, John Pettibone ${ }^{1}$, Jingyu Liu ${ }^{1}$, Aaron Johnston-Peck ${ }^{1}$, Jan Ilavsky ${ }^{2}$

\author{
${ }^{1}$ Material Measurement Lab., National Institute Of Standards \& Technology (NIST), Gaithersburg, United States, ${ }^{2} X$-ray Science \\ Division, Argonne National Laboratory, Argonne, United States \\ E-mail: andrew.allen@nist.gov
}

Silver nanoparticles (AgNPs) have become one of the most extensively used engineered nanomaterials in consumer products and biomedical applications. This is largely due to their capability to slowly release antibacterial Ag+ ions. Rising demand for AgNPs, especially in healthcare applications, is projected to drive the global AgNP market to approach USD 2.5 billion over the next 5 years. However, with increased usage of AgNPs, their inevitable release into the environment raises both health and environmental concerns. Several studies have sought to evaluate the potential environmental outcomes of released AgNPs. Investigations of AgNP sulfidation have particularly drawn attention because of the prevalence of sulfidation in aquatic environments [1]. However, mechanistic details of the reaction pathway transforming AgNPs to silver sulphide (Ag2S) remain controversial topics with conflicting results being reported [1], [2], and knowledge regarding the sulfidation kinetics is limited. New in situ studies by the authors have sought to elucidate the fundamental mechanisms associated with the aggregation behavior, sulfidation and crystallization kinetics, by combining high-resolution synchrotron ultra-small-angle X-ray scattering (USAXS), small angle X-ray scattering (SAXS), and wide-angle X-ray scattering (WAXS) diffraction measurements focused on a well-controlled sulfidation process for polyvinylpyrrolidone (PVP)-coated monodisperse AgNP suspensions in the presence of fulvic acid. These combined techniques simultaneously monitor a length-scale range from sub-angstroms to several micrometers [3], hence allowing a quantitative characterization of the evolution in both the atomic structure and the nanoparticle suspension morphology (microstructure). Here, the in situ measurements have been complemented by ex situ high-resolution scanning transmission electron microscopy (STEM) of the AgNPs previously subjected to the same sulfidation history. Under the real-time experimental conditions used, aggregation of the AgNPs was found to be minimal with the AgNPs remaining spherical, or at least globular throughout the sulfidation process. The incorporation of sulfur ions into the AgNPs causes a monotonic increase in the mean diameter of the nanoparticles, and the nanoparticles exhibit well-defined growth kinetics. It is found that the degree of sulfidation is directly related to the availability of sulfur ions in solution, and the crystallization kinetics of Ag2S correlate well with the growth kinetics of the nanoparticles. Significantly, it was also found that, within the resolution of our X-ray measurements, $\mathrm{Ag}$ ions are not leached into the solution during the sulfidation process.

[1] Thalmann, B., Voegelin, A., Morgenroth, E. \& Kaegi, R. (2016). Environ. Sci.: Nano, 3, 203-212.

[2] Pettibone, J.M. \& Liu, J. (2016). Environ. Sci. Technol., 50, 11145-11153.

[3] Martin, N.M., Allen, A.J., MacCuspie, R.I. \& Hackley, V.A. (2014). Langmuir, 30, 11442-11452.

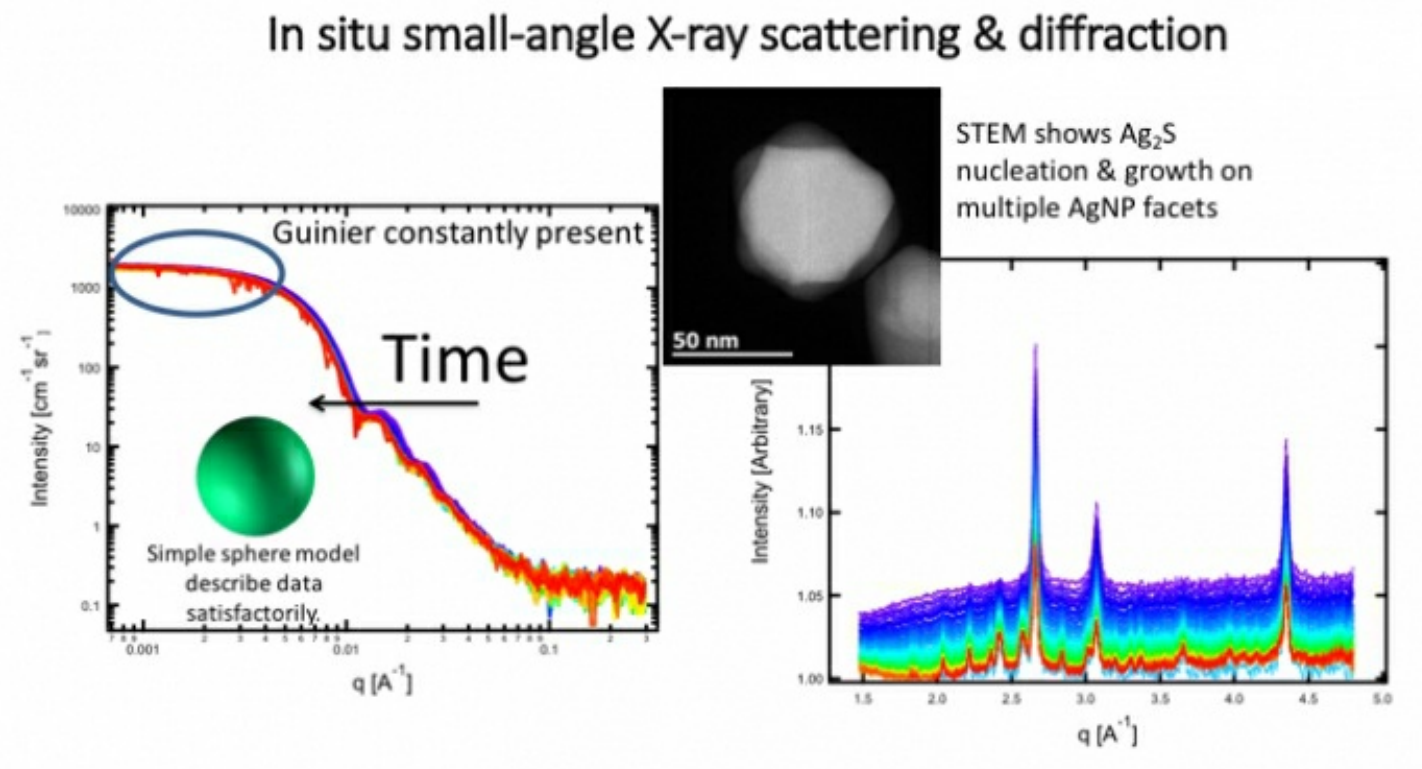

Keywords: silver nanoparticles, in situ reaction kinetics, small-angle X-ray scattering 